\title{
L'HOMME L'Homme
}

Revue française d'anthropologie

160 | octobre-décembre 2001

Droit, coutume, mémoire

Dominique Casajus, Gens de paroles. Langage, poésie et politique en pays touareg

Paris, Éditions La Découverte, 2000, 190 p., annexe, bibl. (« Texte à

l'appui »)

\section{Sophie Caratini}

\section{(2) OpenEdition}

Journals

Édition électronique

URL : http://journals.openedition.org//homme/7863

DOI : 10.4000//homme.7863

ISSN : 1953-8103

Éditeur

Éditions de l'EHESS

Édition imprimée

Date de publication : 1 janvier 2001

Pagination : 249-252

ISBN : 2-7132-1391-6

ISSN : 0439-4216

Référence électronique

Sophie Caratini, « Dominique Casajus, Gens de paroles. Langage, poésie et politique en pays touareg », L'Homme [En ligne], 160 | octobre-décembre 2001, mis en ligne le 31 mai 2007, consulté le 24 septembre 2020. URL : http://journals.openedition.org//homme/7863 ; DOI : https://doi.org/10.4000/ Ihomme.7863

Ce document a été généré automatiquement le 24 septembre 2020.

(c) École des hautes études en sciences sociales 


\section{Dominique Casajus, Gens de paroles. Langage, poésie et politique en pays}

\section{touareg}

Paris, Éditions La Découverte, 2000, 190 p., annexe, bibl. (« Texte à

l'appui »)

\section{Sophie Caratini}

1 À L'INSTAR de Claude Lefort, Dominique Casajus définit sa pratique de recherche comme une «intention de connaissance» et s'oppose vigoureusement à ce qu'il nomme «le programme d'autocontemplation qu'une certaine ethnologie postmoderne veut promouvoir» (p.15). Cette «intention », qui relativise le pouvoir de la science, n'en renie pas le rêve: atteindre l'autre dans sa vérité reste son utopie. La quête de l'impossible s'affirme donc comme source du désir, et ses œuvres, provisoires par essence, fondent leur pertinence sur cet inachèvement.

2 Le chercheur s'énonce comme médiateur, puisqu'il manifeste son "intention de connaissance » dans un double processus d'acquisition et de transmission. Ce faisant, il s'interroge sur l'entreprise de traduction - on pourrait même parler d'interprétation au sens de l'herméneutique - que tout ethnologue est amené à construire lorsqu'il tente de témoigner dans sa langue de l'existence d'une autre culture, des manières d'être, de dire et de penser des hommes qui la partagent. Quoiqu'il en dise, l'auteur n'échappe donc pas complètement à la posture autoréflexive dont il dénonce les excès.

Dominique Casajus nous livre ici la synthèse de ses études et réflexions sur la parole dans le monde touareg, par la présentation et l'analyse de ses usages et de la "vision " (terme qu'il préfère à celui de "représentations ») que les intéressés en donnent. Je ne le suivrai pas complètement lorsqu'il considère que "la matière première de nos travaux d'ethnologues n'est rien d'autre que la parole de ceux qui nous accueillent et nous accordent l'hospitalité durant ce que nous appelons nos années de terrain" (p. 11), et nuancerai sans doute l'affirmation selon laquelle « ceux qui nous parlent ne nous demandent pas d'interpréter leurs dires, mais simplement de les écouter» (p. 12). Il me semble en effet que la connaissance acquise sur le terrain, pour une grande part 
transmise par ceux qui nous reçoivent, va bien au-delà du simple recueil de paroles, et que l'enjeu, pour eux, des discours qu'ils nous tiennent ou de ce qu'ils nous donnent à voir et à comprendre ne se réduit pas non plus au simple plaisir d'être écoutés par celui qui passe. Informés de l'objectif de publication que nous nous sommes donné, conscients de l'importance de l'écrit dans le monde contemporain et en particulier dans les pays dominants, il me paraît au contraire que les nomades sahariens pourraient avoir aussi comme but, lorsqu'ils nous " parlent », de nous utiliser comme... porte-parole, quels que soient les liens personnels liés par ailleurs en cette occasion. Néanmoins, lorsque l'auteur met sur le devant de la scène la parole recueillie plutôt que la sienne et tente de faire accéder le lecteur le plus directement possible aux dires des Touaregs, il donne à son livre une valeur double: d'une part, il fournit des données susceptibles d'ouvrir le champ de la comparaison bien plus efficacement que l'argumentaire qui les occulte trop souvent; d'autre part, il rend aux Touaregs le don de l'hospitalité en transportant concrètement leur parole au-delà de leurs frontières. Mais il ne s'agit pas pour autant d'une livraison de données brutes, et il était difficile au chercheur de trouver l'équilibre entre la restitution des discours recueillis et ses propres interventions. Le livre apparaît comme une réflexion très construite, qui cherche à valoriser son objet sans pour autant s'effacer derrière lui. C'était une gageure, sans doute, que de vouloir mettre en exergue la parole des Touaregs et de la rendre en même temps accessible au lecteur. Ce faisant, Dominique Casajus a-t-il réussi à ne pas « interpréter » ? La question reste ouverte...

L'ouvrage se compose d'une introduction suivie de huit parties qui sont autant d'entrées dans cette parole touarègue, une méthode délibérément plurielle qui s'adapte à la richesse et à la diversité d'un langage dont l'expression ne saurait se réduire à quelques grandes catégories descriptives, occidentales de surcroît. De même qu'il passe d'un lieu de cette parole à un autre, l'auteur se donne la liberté de glisser lui aussi d'une écriture à l'autre, d'un type de commentaire à un autre, cherchant à s'adapter chaque fois aux diverses facettes de cet objet autour duquel il tourne, multipliant les éclairages, entraînant le lecteur dans une sorte de promenade linguistique dont il est à la fois le guide et le pédagogue. Le cheminement est sinueux, parfois hétérogène, mais dans l'ensemble l'effet est réussi. Le livre est agréable à lire comme à parcourir, et permet indubitablement d'accéder à quelque chose d'important en ce qu'il ouvre l'esprit à cette autre "intention de connaissance» qui est, sinon de "comprendre», du moins d'entendre pourquoi les Touaregs se disent " gens de la parole». En ce sens, et quelles que soient les critiques qu'on pourrait formuler çà et là, Dominique Casajus nous donne ici un de ces livres rares qui « parviennent à trouver les mots justes pour dire ce que les hommes vivent » (p. 15).

5 L'introduction, classique, décrit succinctement le monde touareg à travers quelques données élémentaires parmi lesquelles sont privilégiés les noms : noms de lieux, noms de fractions - le paysage et l'organisation sociale apparaissent tels que les Touaregs les désignent. Ce qui fait pour eux référence est mis en regard avec ce qu'en ont écrit les quelques voyageurs et historiens passés par là depuis le XIve siècle, à quoi s'ajoutent des informations sur les rebellions du Mali et du Niger des années 1990, ainsi que sur la place du critère linguistique dans les revendications politiques. Ce rapide survol permet à l'auteur d'articuler son travail sur la question identitaire telle qu'elle est posée par la jeunesse touarègue contemporaine, inquiète de reconstruire son histoire et surtout de définir sa culture, une jeunesse que Dominique Casajus voudrait pouvoir rassurer, et à laquelle s'adresse pour une grande part sa démonstration. En ce sens, la posture 
critique du chercheur en regard du postmodernisme se traduit aussi par la volonté d'établir un véritable dialogue avec ceux dont il a fait son objet d'étude.

6 La question de l'identité touarègue est abordée dès le premier chapitre, intitulé "Les gens de la parole ", qui présente la vision locale du statut de la parole et de la langue et analyse son rôle dans les constructions identitaires passées et présentes. Montrant que «ce par quoi ils se distinguent des autres, ce n'est pas seulement leur langue, mais le fait qu'elle est le trait par lequel ils choisissent de s'en distinguer» (p. 26), l'auteur conclut sur cette idée que les Touaregs ont, plus que d'autres, le «souci» de leur parole. Ce faisant, il fait l'hypothèse que l'identité touarègue doit être davantage recherchée dans ce « souci » qui conditionne l'usage que dans l'usage lui-même.

7 Les deux chapitres suivant, «La parole pénombreuse » et "L'enfant et les sortilèges ", saisissent, à travers l'exemple de la vie quotidienne, la place particulière du dit et du non-dit dans cette société où le summum de l'éducation - et de la " noblesse » - est la suggestion. Le fait ne doit pas être étalé, le mot ne doit pas être prononcé, tout l'art du dire chez les Touaregs " bien nés » réside en l'évitement du signifiant, et en la capacité de l'interlocuteur de deviner, d'entendre ce qui doit être entendu du fait de cette absence. La moquerie, voire la mise en dérision du "grossier personnage » désigne ici non pas celui qui ne comprend pas le dit, mais celui qui ne sait pas interpréter correctement le silence. En quoi le «souci » touarègue de la parole révèle la part importante que prend la communication non verbale dans cette culture, une part qu'on aurait aimé que Dominique Casajus développe davantage. En revanche, on retiendra, en regard de la valeur du non-dit dans ces sociétés, l'intérêt de l'évocation des "êtres de silence ", ou kel-esuf, dont l'imaginaire des nomades peuple les solitudes qui les entourent : "Vivant à la lisière des espaces habités, les kel-esuf se tiennent aussi sur les lisières de la parole, lesquelles peuvent être franchies à la fois par l'excès et par le manque. Le manque [...] c'est le silence des déments et des muets, l'anonymat du nouveau-né qui ne sait que vagir, peut-être encore l'oubli où lentement, quand leur nom s'est perdu, sombrent les trépassés » (p. 63).

Deux chapitres sont consacrés à la poésie : le chapitre III, «Le poète et le silence ", et le chapitre V, «Art poétique et art de la guerre». Entre les deux, le chapitre IV, «La parole et la noblesse ", fait passer le lecteur de l'individu (le poète) au groupe (la guerre et la figure de l'autre). Comme dans la plupart des sociétés, l'art est espace de transgression. Le poète va donc dire ce qui ne se dit jamais : le désir, l'amour, la beauté, l'espoir, la souffrance, la peur, la folie et la mort. Traversant les différents genres poétiques, les principaux thèmes de la poésie touarègue, même lorsqu'ils sont universels, reflètent ce que le désert et la vie nomade imposent à chacun : la séparation, l'absence, la solitude. Le manque, et la souffrance causée par le manque, dominent dans la plupart des textes cités. La parole du poète, émise dans la solitude, nie l'absence de l'aimée, lui donne réalité, réfute le silence. En contrepoint, la voix de la femme poète chante l'inquiétude et la mélancolie de celle qui reste immobile, enfermée dans l'attente. Seul vécu d'un désir poussé à son paroxysme par un trop de manque, d'absence et de silence, la poésie des nomades exalte celui qui l'émet au point de le porter au bord de la folie. Aussi Dominique Casajus écrit-il en conclusion: "D'une manière comparable, lorsqu'il erre au-delà des lisières de la parole ordinaire, le poète touareg rappelle la profondeur des abîmes sur lesquels s'énonce toute parole » (p. 91).

$9 \quad$ Fil qui enserre les vivants, les protégeant du monde des morts, cordage jeté au-dessus du vide et qui relie les amants séparés, la parole touarègue préserve également les 
hommes de l'errance en attribuant à chacun une place dans l'espace social. La honte et le déshonneur sont des sentiments très vifs qui risquent à tout instant de s'emparer de celui qui manque à sa parole ou défaille dans l'art de s'adresser à son prochain. À chaque sexe, chaque rang, chaque âge, presque chaque lignage, et d'une manière générale à chaque statut social est un mode convenu de s'exprimer. Un mode qui s'acquiert et qui se repère, et dont le plus valorisé est celui du "noble ». Si le terme de "noble ", attribué par les militaires français aux élites touarègues (et repris par les chercheurs), a pu paraître obscur tant il paraît chargé de références occidentales, Dominique Casajus propose dans son chapitre $\mathrm{V}$ une appréhension à la fois originale et très éclairante de ce que peut vouloir dire « être noble » chez les Touaregs, en regard de la question de la reproduction sociale. Dépassant les analyses économiques ou politiques qui ont pu être faites par les anthropologues ou les historiens de la "noblesse " touarègue, il fait voir qu'à la source de la reconnaissance du noble se trouve une manière de "parler » qui est aussi une manière d'être et de se comporter que l'on pourrait rapporter à l'idée de "style». Si l'on peut regretter que l'auteur n'aille pas jusqu'à contester l'usage du terme par trop archaïque de «noble», on appréciera l'importance de son apport pour la compréhension des évolutions actuelles non seulement de la société touarègue mais de la plupart des sociétés, sousdéveloppées ou non.

10 L'usage de l'art poétique dans la guerre illustre ce rôle de la parole dans la répartition du soi et de l'autre au sein de l'espace physique et social. Dominique Casajus montre comment les Touaregs se situent les uns par rapport aux autres, que ces autres soient proches, " mêmes », ou lointains, " étrangers », et comment l'art de s'adresser ou de ne pas s'adresser à l'ennemi marque les frontières mentales des identités collectives, définit l'altérité. La violence, rapport social dominant des groupes nomades du désert saharien, est donc socialisée à l'extrême, et s'exprime non seulement dans le langage des armes mais dans celui du verbe. Un verbe codifié dont l'absence est un des signifiants possibles.

11 Dans le chapitre VII, intitulé « Le sultan d'Agadez », l'auteur revient sur le thème de la noblesse, mais cette fois au niveau de l'organisation politique. En rupture avec le style des précédents chapitres, l'histoire du sultanat telle qu'elle est ici rapportée apparaît un peu décalée par rapport au propos général en ce sens que l'analyse ne s'appuie plus sur la « parole » touarègue. Il était néanmoins important de poser la question de l'islam dans le système politique touarègue, et de montrer quelles fonctions il remplit dans l'institution sultanale, la désignant non pas comme lieu du pouvoir mais de l'arbitrage. Toutefois, je ne partage pas le point de vue de Dominique Casajus lorsqu'il affirme que "l'institution sultanale ressortit à une autre logique que celle de la tâmmujegha (noblesse ou targuité) »(p.144), car j'ai tendance à penser que les contradictions relevées sont les termes dialectiques de ce qui fonde le système qu'il décrit. Si l'incompatibilité qu'il remarque entre les valeurs d'égalité prônées par l'islam et les fondements hiérarchiques de la société touarègue alimente aujourd'hui les clivages internes, il semble que cette « faiblesse » contemporaine du système lui ait au contraire donné toute sa force dans le passé.

Dans le dernier chapitre, «Le poète et le lettré », l'auteur aborde la question essentielle du rapport entre le statut de la langue touarègue et celui de la langue arabe. Ce thème, universel à l'échelle du monde musulman dont une grande partie n'est pas arabophone, vaut également pour les pays arabes dans lesquels la langue en usage est toujours 
différente de celle des lettrés. Langue de Dieu, l'arabe classique apparait comme ce que les psychanalystes qualifieraient de "fétichisme du signifiant». Dans le cas des "gens de parole", il y a donc là, inévitablement, une confrontation douloureuse entre ces deux fétichismes, entre un profane sacralisé et un sacré qui lui fait concurrence, imposant d'incessantes négociations entre Dieu et le Diable, et entre soi et soi.

\section{AUTEUR}

SOPHIE CARATINI

Laboratoire URBAMA, Université de Tours, Tours. 